\title{
SUSAR Reporting to EudraVigiliance Clinical Trial Module Indicator
}

National Cancer Institute

\section{Source}

National Cancer Institute. SUSAR Reporting to EudraVigiliance Clinical Trial Module

Indicator. NCI Thesaurus. Code C126073.

An indication as to whether suspected unexpected serious adverse reactions will be reported to a EudraVigiliance clinical trial module. 